\title{
Chaos Suppression in NEMs Resonators by Using Nonlinear Control Design
}

\author{
Angelo Marcelo Tusset ${ }^{1}$, Atila Madureira Bueno ${ }^{2}$, Claudinor Bitencourt \\ Nascimento $^{3}$, Mauricio dos Santos Kaster ${ }^{4}$, José Manoel Balthazar ${ }^{5}$ \\ ${ }^{1}$ UTFPR,Ponta Grossa,Brazil, tusset@utfpr.edu.br; ${ }^{2}$ UTFPR,Ponta Grossa,Brazil, atilabueno@utfpr.edu.br; \\ ${ }^{3}$ UTFPR, Ponta Grossa,Brazil, claudinor@utfpr.edu.br; ${ }^{4} U T F P R$, Ponta Grossa,Brazil,_mkaster@utfpr.edu.br; \\ ${ }^{5}$ UNESP, Rio Claro, Brazil, jmbaltha@rc.unesp.br
}

\begin{abstract}
In this work the chaotic behavior of a micro-mechanical resonator with electrostatic forces on both sides is suppressed. The aim is to control the system in an orbit of the analytical solution obtained by the Method of Multiple Scales. Two control strategies are used for controlling the trajectory of the system, namely: State Dependent Riccati Equation (SDRE) Control and Optimal Linear Feedback Control (OLFC). The controls proved effectiveness in controlling the trajectory of the system. Additionally, the robustness of each strategy is tested considering the presence of parametric errors and measurement noise in control.
\end{abstract}

Keywords: Optimal Control, SDRE Control, Chaos, MEMS.

PACS: 05.45.-a

\section{INTRODUCTION}

Currently a great deal of research has been performed to report chaotic behavior in MEMS resonators $[1,2]$. In order to drive the chaotic movement to a stable orbit, an Optimal Linear Feedback Control (OLFC) is used in [3], SDRE Control is used in [4] Robust Adaptive Fuzzy Control in [5], Fuzzy Sliding Mode Control Design in [6].

The SDRE strategy first proposed by [7] and later expanded by [8], was independently studied by [9] and alluded to by [10]. The SDRE strategy is an effective algorithm for synthesizing nonlinear feedback controls by allowing nonlinearities in the system states, while additionally offering great design flexibility through state-dependent weighting matrices [11].

The Optimal Linear Feedback Control was proposed by [12]. In [12] the quadratic nonlinear Lyapunov function was proposed to resolve the optimal nonlinear control design problem. The theorem formulated by [12] explicitly expresses the form of minimized functional and gives the sufficient conditions that allow using the Linear Feedback Control for nonlinear systems [13, 14].

The micromechanical resonator system studied in this work is depicted in Figure 1. Considering the device of Figure 1 as consisting of two fixed plates and a movable plate between them, to which is applied a voltage $V(t)$ composed of a polarization voltage (DC) $V_{p}$, and alternating voltage $(A C) V_{i} \sin (\omega t)$.

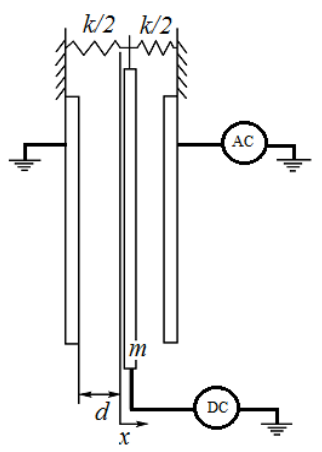

FIGURE 1. Micromechanical resonator.

where: $d$ (distance between the plates), $x$ (lateral movement), $m$ (front panel mass), $D C$ (polarization voltage $V_{p}$ ) and $A C$ (alternating voltage $V_{i} \sin (\omega t)$ ).

\section{MATHEMATICAL MODEL OF MICRO ELECTROMECHANICAL RESONATOR}

The equation of motion of the plates is given by:

$$
m \ddot{x}=-F_{k}-F_{c}+F_{e}
$$

where: $F_{k}$ is the conservative force of the spring, $F_{c}$ the damping force of the elastic term and $F_{e}$ the electric force. 
According to [4] the forces $F_{k}$ and $F_{c}$ can be defined as:

$$
\begin{gathered}
F_{k}=k_{1} x+k_{3} x^{3} \\
F_{c}=c \dot{x}
\end{gathered}
$$

According to $[5,15]$ the force $F_{e}$ can be defined as:

$$
F_{e}=\frac{1}{2} \frac{C_{0}}{(d-x)^{2}}\left(V_{p}+V_{i} \sin (\omega t)\right)-\frac{1}{2} \frac{C_{0}}{(d-x)^{2}} V_{p}^{2}
$$

where: $C_{0}$ (capacitance of the parallel-plate actuator).

Substituting (2), (3) and (4) in (1) we obtain the equation of motion:

$$
\begin{aligned}
& m \ddot{x}+k_{1} x+k_{3} x^{3}+c \dot{x}= \\
& +\frac{1}{2} \frac{C_{0}}{(d-x)^{2}}\left(V_{p}+V_{i} \sin (\omega t)\right)-\frac{1}{2} \frac{C_{0}}{(d-x)^{2}} V_{p}^{2}
\end{aligned}
$$

According to [5] the equation (5) can be represented in nondimensional form:

$$
\begin{aligned}
& \ddot{u}+\mu \dot{u}+u+\alpha_{3} u^{3}=\gamma\left(\frac{1}{(1-u)^{2}}-\frac{1}{(1+u)^{2}}\right) \\
& +\frac{\sigma}{(1-u)^{2}} \sin (w T)
\end{aligned}
$$

where: $\quad w=\frac{\omega}{\omega_{0}}, \quad T=\omega_{0} t, \quad \omega_{0}^{2}=\frac{k_{1}}{m}, \quad u=\frac{x}{d}$, $\mu=\frac{c}{m \omega_{0}}, \alpha_{3}=\frac{k_{3} d^{2}}{m \omega_{0}^{2}}, \gamma=\frac{C_{0} \mathrm{~V}_{\mathrm{p}}^{2}}{2 m \omega_{0}^{2} d^{3}}$, and $\sigma=\frac{2 \gamma V_{i}}{V_{p}}$.

Rewriting equation (6) in state space:

$$
\begin{aligned}
& y_{1}^{\prime}=y_{2} \\
& y_{2}^{\prime}=-\mu y_{2}-y_{1}-\alpha_{3} y_{1}^{3}+\gamma\left(\frac{1}{\left(1-y_{1}\right)^{2}}-\frac{1}{\left(1+y_{1}\right)^{2}}\right) \\
& +\frac{\sigma}{\left(1-y_{1}\right)^{2}} \sin (w T)
\end{aligned}
$$

where: $y_{1}=u$ and $y_{2}=\dot{u}$.

In Figure 2, the displacement, the phase portrait diagram, the Lyapunov exponent and the Poincare map are shown considering the parameters: $\mu=0.01$, $\alpha_{3}=12, \gamma=0.338, \sigma=0.03558$ and $w=0.5$.

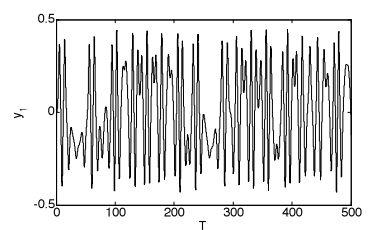

(a)

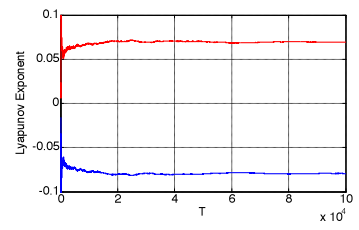

(c)

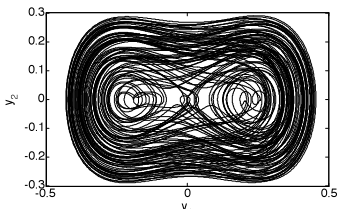

(b)

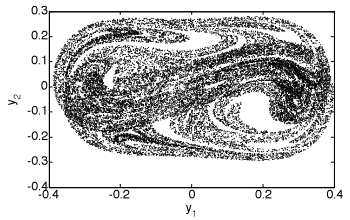

(d)
FIGURE 2. (a): The displacement of AFM without control. (b): Phase portrait of atomic force microscope. (c):

Exponents of Lyapunov: $\lambda_{1}=0.0694$ and $\lambda_{2}=-0.0794$.

(d): Poincare map.

As can be observed in Figure 2c, the system has a positive Lyapunov exponent. The chaotic behavior also can be observed in the phase portrait in Figure $4 \mathrm{~b}$ and Poincare map in Figure 4d.

\section{ANALYTICAL APPROXIMATE SOLUTIONS OBTAINED THROUGH THE PERTURBATION METHOD}

This procedure is used in order to obtain an approximate analytical solution [16]. Considering first the rational substitution of the term of the equation (6):

$\left[\frac{1}{(1-u)^{2}}-\frac{1}{(1+u)^{2}}\right]$ and $\frac{1}{(1-u)^{2}}$ by a polynomial function: $P_{4}(u)=\delta_{0}+\delta_{1} u+\delta_{2} u^{2}+\delta_{3} u^{3}+\delta_{4} u^{4}$, where $-0.5 \leq u \leq 0.5$.

According to [17] one can approximate the two functions by least squares method minimizing the error:

$$
\int_{-0.5}^{0.5}\left[(f(u))-\left(P_{4}(u)\right)\right] d u
$$

Resulting in the following approximation:

$$
\begin{aligned}
& {\left[\frac{1}{(1-u)^{2}}-\frac{1}{(1+u)^{2}}\right]=0.0674 u^{2}+11.0263 u^{3}} \\
& \frac{1}{(1-u)^{2}}=0.9585+1.8525 u+4.498 u^{2}+6.426 u^{3}(
\end{aligned}
$$

Substituting (9) and (10) in (6) we obtain the following differential equation: 


$$
\begin{aligned}
& \ddot{u}+\mu \dot{u}+u+a_{2} u^{2}+a_{3} u^{3}=a_{4} u^{3} \sin (w T) \\
& +a_{5} u^{2} \sin (w T)+a_{6} u \sin (w T)+f \sin (w T)
\end{aligned}
$$

where: $\quad a_{2}=-0.0674, \quad a_{3}=0.9737, \quad a_{4}=0.2286$, $a_{5}=0.1772, a_{6}=0.0659, f=0.0341$ and $\mu=0.01$.

Now, we will use the method of multiple scales to find analytically an approximate analytical solution to the above governing equation, this is done for a balance of order as follows. Therefore the equation is:

$$
\begin{aligned}
& \ddot{u}+u+\varepsilon \mu \dot{u}+\varepsilon^{2} a_{2} u^{2}+\varepsilon a_{3} u^{3}=\varepsilon^{2} a_{4} u^{3} \sin (w T) \\
& +\varepsilon^{2} a_{5} u^{2} \sin (w T)+\varepsilon^{2} a_{6} u \sin (w T)+f \sin (w T)
\end{aligned}
$$

Where $\varepsilon$ is the parameter responsible for this balance [16]. Introducing the scales $T_{0}=T$ and $T_{1}=\varepsilon T$. Seeking solutions in the following way:

$$
u=u_{0}\left(T_{0}, T_{1}\right)+\varepsilon u_{1}\left(T_{0}, T_{1}\right)+\ldots
$$

As the original independent variable (time scale $T$ ) was substituted by independent scales $T_{0}$ and $T_{1}$, derivatives with respect to $T$ should be expressed in terms of partial derivatives in respect of $T_{n}$ such that:

$$
\begin{aligned}
& \frac{d}{d T}=D_{0}+\varepsilon D_{1}+\ldots \\
& \frac{d^{2}}{d T^{2}}=D^{2}{ }_{0}+2 \varepsilon D_{0} D_{1}+\ldots
\end{aligned}
$$

Substituting (13) in (12) and considering the derivatives (14), (12) is represented in form perturbed:

$$
\begin{aligned}
& \left(D^{2}{ }_{0}+2 \varepsilon D_{0} D_{1}\right)\left(u_{0}+\varepsilon u_{1}\right)+\varepsilon \mu\left(D_{0}+\varepsilon D_{1}\right)\left(u_{0}+\varepsilon u_{1}\right) \\
& +\left(u_{0}+\varepsilon u_{1}\right)+\varepsilon^{2} a_{2}\left(u_{0}+\varepsilon u_{1}\right)^{2}+\varepsilon a_{3}\left(u_{0}+\varepsilon u_{1}\right)^{3} \\
& -\varepsilon^{2} a_{4}\left(u_{0}+\varepsilon u_{1}\right)^{3} \sin \left(w T_{0}\right)-\varepsilon^{2} a_{5}\left(u_{0}+\varepsilon u_{1}\right)^{2} \sin \left(w T_{0}\right) \\
& -\varepsilon^{2} a_{6}\left(u_{0}+\varepsilon u_{1}\right) \sin \left(w T_{0}\right)-f \sin \left(w T_{0}\right)=0
\end{aligned}
$$

Separating the terms in relation with the potential for $\varepsilon^{0}$ and $\varepsilon^{1}$ we have:

$$
\begin{gathered}
\varepsilon^{0}: D_{0}^{2} u_{0}+u_{0}=f \sin \left(w T_{0}\right) \\
\varepsilon^{1}: D_{0}^{2} u_{1}+u_{1}=-2 D_{0} D_{1} u_{0}-\mu D_{0} u_{0}-a_{3} u_{0}^{3}
\end{gathered}
$$

where:

$$
u_{0}=A\left(T_{1}\right) e^{i T_{0}}+\Lambda e^{i w T_{0}}+c c
$$

$$
A=\frac{1}{2} a e^{i \beta} \text { and } \Lambda=\frac{f}{2\left(1-w^{2}\right)}
$$

substituting (18) in (17) we obtain:

$$
\begin{aligned}
& \left.D_{0}^{2} u_{1}+a u_{1}=-i\left(2 A^{\prime}+\mu A\right) e^{i T_{0}}+i\left(2 \bar{A}^{\prime}+\mu \bar{A}\right)\right)^{-i T_{0}} \\
& -i \mu w e^{i w T_{0}}+i \mu w e^{-i w T_{0}} \\
& -a_{3}\left[A\left(T_{1}\right) e^{i T_{0}}+\Lambda e^{i w T_{0}}+\bar{A}\left(T_{1}\right) e^{-i T_{0}}+\bar{\Lambda} e^{-i w T_{0}}\right]
\end{aligned}
$$

Eliminating the secular terms, of the equation (20) as follows:

$$
2 i A^{\prime}+\mu i A+3 a_{3}\left(A \bar{A}+2 A^{2}\right) A=0
$$

Substituting (19) into (21) and separating real and imaginary parts gives:

$$
\left\{\begin{array}{l}
a^{\prime}=0 \\
\beta^{\prime}=\frac{3 a_{3} a^{2}}{8}+\frac{3 a_{3} a^{2}}{2} \cos (2 \beta)
\end{array}\right.
$$

$\beta$ is calculated numerically integrating equation (22). One possible solution for $u$ is:

$$
u=a \cos (T+\beta)+\frac{4 f}{3} \sin (w T)
$$

where: $\quad a=0.2, \quad \beta=0.9117, \quad f=0.0341 \quad$ and $w=0.5$.

\section{NONLINEAR CONTROL DESIGN}

The objective is to determine a signal control $U$, which carries the system (6) from any initial state to final state:

$$
e(\infty)=0
$$

where:

$$
e=\left[\begin{array}{ll}
y_{1}-\tilde{y}_{1} & y_{2}-\tilde{y}_{2}
\end{array}\right]
$$

Where $\tilde{y}$ is the vector of desired orbits.

one possible solution for (16) in polar form is: 


\section{Application of Optimal Linear Feedback Control}

The equations that describe the motion of the system with the control law $U$ are described by the following nonlinear equations:

$$
\begin{aligned}
& y_{1}^{\prime}=y_{2} \\
& y_{2}^{\prime}=-\mu y_{2}-y_{1}-\alpha_{3} y_{1}^{3} \\
& +\frac{\left(4 \gamma+2 \sigma \sin (w t)+y_{1} \sigma \sin (w t)\right) y_{1}}{\left(1-y_{1}^{2}\right)^{2}} \\
& +\frac{\sigma}{\left(1-y_{1}^{2}\right)^{2}} \sin (w t)+U
\end{aligned}
$$

with:

$$
U=\tilde{u}_{o}+u_{o f}
$$

Where $u_{o f}$ is the feedback control, and $\tilde{u}_{o}$ is the feedforward control, for optimal control, given by:

$$
\begin{aligned}
& \tilde{u}_{0}=\tilde{y}_{2}{ }_{2}+\mu \widetilde{y}_{2}+\tilde{y}_{1}+\alpha_{3} \tilde{y}_{1}^{3} \\
& -\frac{\left(4 \gamma+2 \sigma \sin (w T)+\tilde{y}_{1} \sigma \sin (w T)\right) \tilde{y}_{1}}{\left(1-\tilde{y}_{1}^{2}\right)^{2}}-\frac{\sigma}{\left(1-\tilde{y}_{1}^{2}\right)^{2}} \sin (w T)
\end{aligned}
$$

Replacing (28) into (26) and considering the deviations (25) we obtain:

$$
\begin{aligned}
& e_{1}^{\prime}=e_{2} \\
& e_{2}^{\prime}=-\mu e_{2}-e_{1}-\alpha_{3}\left(y_{1}-\tilde{y}_{1}\right)^{3}+\alpha_{3} \tilde{y}_{1}^{3} \\
& +\frac{\left(4 \gamma+2 \sigma \sin (w T)+\left(y_{1}-\tilde{y}_{1}\right) \sigma \sin (w T)\right)\left(y_{1}-\tilde{y}_{1}\right)}{\left(1-\left(y_{1}-\tilde{y}_{1}\right)^{2}\right)^{2}} \\
& -\frac{\left(4 \gamma+2 \sigma \sin (w T)+y_{1} \sigma \sin (w T)\right)_{1}}{\left(1-\tilde{y}_{1}^{2}\right)^{2}} \\
& +\frac{\sigma}{\left(1-\left(y_{1}-\tilde{y}_{1}\right)^{2}\right)^{2}} \sin (w T)-\frac{\sigma}{\left(1-\tilde{y}_{1}^{2}\right)^{2}} \sin (w T)+u_{o f}
\end{aligned}
$$

Considering the system (29) written in the following way:

$$
e^{\prime}=A e+G(e, \tilde{y})+B u_{o f}
$$

where:

$e=\left[\begin{array}{l}e_{1} \\ e_{2}\end{array}\right], A=\left[\begin{array}{cc}0 & 1 \\ -1 & -\mu\end{array}\right], B=\left[\begin{array}{l}0 \\ 1\end{array}\right]$ and

$$
G(e, \tilde{y})=\left[\begin{array}{l}
0 \\
\left.\frac{-\alpha_{3}\left(y_{1}-\tilde{y}_{1}\right)^{3}+\alpha_{3} \tilde{y}_{1}^{3}+}{\left(\frac{4 \gamma}{\sigma}+\sin (w T)\left(2+\left(y_{1}-\tilde{y}_{1}\right)\right)\right.}\right) \sigma\left(y_{1}-\tilde{y}_{1}\right) \\
\left(1-\left(y_{1}-\tilde{y}_{1}\right)^{2}\right)^{2} \\
-\frac{\left(4 \gamma+2 \sigma \sin (w T)+y_{1} \sigma \sin (w T)\right) \tilde{y}_{1}}{\left(1-\tilde{y}_{1}^{2}\right)^{2}} \\
+\left(\frac{\sigma}{\left(1-\left(y_{1}-\tilde{y}_{1}\right)^{2}\right)^{2}}-\frac{\sigma}{\left(1-\tilde{y}_{1}^{2}\right)^{2}}\right) \sin (w T)
\end{array}\right]
$$

According to [13, 14], if there are an error weighted matrix $Q$, and the control weighted matrix $R$, positive definite symmetric matrix, and a matrix Riccati $P$, such that the matrix:

$$
\widetilde{Q}=Q-G^{T}\left(e, x^{*}\right) P-P G\left(e, x^{*}\right)
$$

is positive definite matrix $G$ restricted, then the control $u_{o f}$ is optimal and transfers the non-linear systems from any initial state, to the final state:

$$
e(\infty)=0
$$

minimizing the functional:

$$
J=\int_{0}^{\infty}\left(e^{T} \widetilde{Q} e+u_{o f}{ }^{T} R u_{o f}\right) d t
$$

Then control $u_{0 f}$ can be found by solving the equation:

$$
u_{o f}=-R^{-1} B^{T} P e=-k e
$$

Since the symmetric matrix $P$, can be obtained from the Riccati algebraic equation:

$$
P A+A^{T} P-P B R^{-1} B^{T} P+Q=0
$$

Using the matrices $A$ and $B$ (31), choosing:

$$
Q=\left[\begin{array}{cc}
10^{2} & 0 \\
0 & 10^{2}
\end{array}\right] \text { and } R=\left[10^{-2}\right]
$$

and using the command $k=\operatorname{lgr}(A, B, Q, R)$ from Matlab $^{\mathrm{r}}$, we get:

$$
u_{o f}=-99.005 e_{1}-100.975 e_{2}
$$


For the optimal control verification (38), the function (32) is numerically calculated with $L(T)=e^{T} \widetilde{Q} e \quad[13,14]$. The next figure shows the trajectory of the periodic function, considering the application of control, and the desired orbit $\left(\tilde{y}_{1}\right)$ the equation (23).

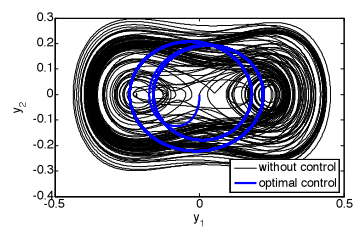

(a)

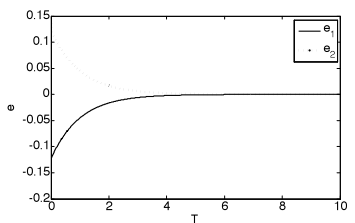

(b)

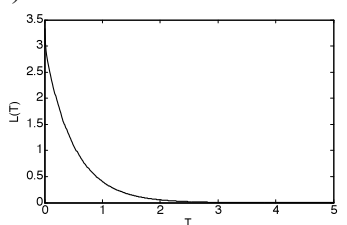

(c)

FIGURE 3. (a): Phase portrait, chaotic (black) and controlled orbit (blue) (b): Signal deviations (c): $L(T)$ calculated in optimal

In Figure 3, it can be seen that the control was effective to move the system from a chaotic state to a periodic orbit (23).

\section{Application SDRE Control}

The dynamic system defined by equation (26) can be parameterized as a first order state equation and written in the state-dependent coefficient (SDC) and non state-dependent coefficient in the following way:

$$
y^{\prime}=A(y) y+B U_{s}+F(y, T)
$$

Where $y=\left[\begin{array}{ll}y_{1} & y_{2}\end{array}\right]$ is state time dependent, $y^{\prime} \in R^{2}$ is the vector of the first order time derivates of the states. $U_{s}=u_{s f}+\widetilde{u}_{s}$, where $u_{s f}$ the feedback control, $\widetilde{u}_{s}$ is the feedforward control, and $F(y, T)$ is the nonlinear vector.

And the complete system by:

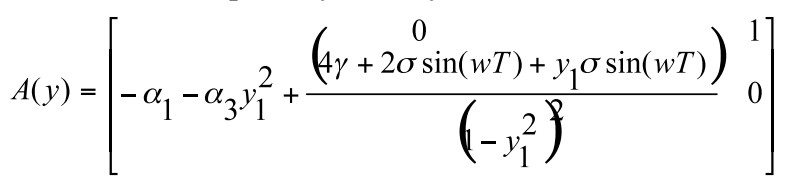
$B=\left[\begin{array}{l}0 \\ 1\end{array}\right]$ and $F(y, T)=\left[\begin{array}{c}0 \\ \frac{\sigma \sin w T}{\left(1-y_{1}^{2}\right)^{2}}\end{array}\right]$
A state feedback instead of output feedback is adopted to enhance the control performance. The cost function for the regulator problem is given by:

$$
J=\frac{1}{2} \int_{t_{0}}^{\infty}\left[y^{T} Q(y) y+u_{s f}^{T} R(y) u_{s f}\right] t
$$

Where $Q(y)$ is semi-positive-definite matrix and $R(y)$ positive definite. Assuming full state feedback, the control law is given by:

$$
u_{s f}=-R^{-1}(y) B^{T}(y) P(y) y=-k(y) y
$$

The estate-dependent Riccati equation to obtain $P(y)$, is given by:

$$
\begin{aligned}
& A^{T}(y) P(y)+P(y) A(y)-P(y) B(y) R^{-1}(y) B^{T}(y) P(y) \\
& +Q(y)=0
\end{aligned}
$$

Defining the feedforward control as:

$$
\tilde{u}_{s}=\left[\begin{array}{c}
0 \\
-\frac{\sigma \sin w T}{\left(1-y_{1}^{2}\right)^{2}}
\end{array}\right]
$$

Replacing (44) into (39), the system (39) can be represented in the form:

$$
y^{\prime}=A(y) y+B u_{s f}
$$

The next figure shows the trajectory of the periodic function considering the application of command $k=\operatorname{lqr}(A, B, Q, R)$, using the matrices $\mathrm{A}$ and $\mathrm{B}(40)$, choosing $Q$ and $R$ (37), and desired orbit $\tilde{y}_{1}$ (23).

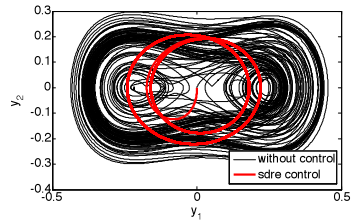

(a)

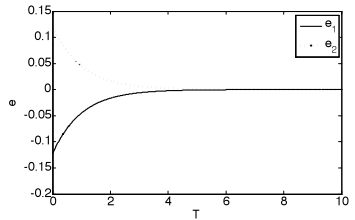

(b)
FIGURE 4. (a): Phase portrait, chaotic (black) and controlled orbit (red). (b): Signal deviations.

We can see in Figure 4, that the SDRE control also was effective to take the system from a chaotic state to a periodic orbit (23). 


\section{Control System Behavior In The Presence Of Parametric Errors And Measurement Noise}

The parameters used in the control were obtained from a data set. The data set provided parametric errors, as measurement errors or model uncertainties. To consider the effect of parameter uncertainties on the performance of the controller $U$, the parameters used in the control will be considered as a random error of $\pm 20 \% \quad[18]$.

To consider the effect of measurement noise on the performance of the controller, a sinusoidal noise with random frequency and amplitude of noise is added:

$$
e_{\text {noise }}=\psi(T) \sin (\varpi T)
$$

$\psi(T)=0.05 \pm 0.002 * r(T), \quad \varpi(T)=0.5 \pm 0.02 * r(T)$ and $r(t)$ are normally distributed random functions.

In Figure 5, we observe the robustness of the control to maintain the system in the desired orbit (23).

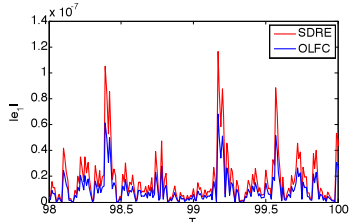

(a)

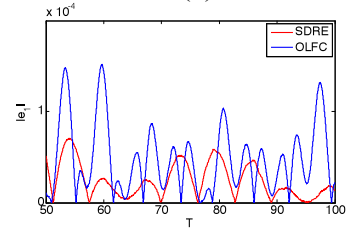

(c)

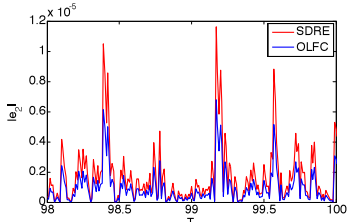

(b)

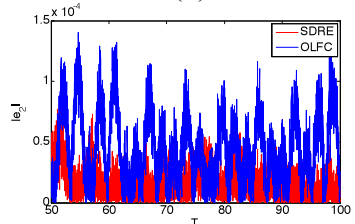

(d)
FIGURE 5. (a): Signal deviations $\left|e_{1}\right|$ without parameter uncertainties and measurement noise, (b): Signal deviations $\left|\mathrm{e}_{2}\right|$ without parameter uncertainties and measurement noise. (c): Signal deviations $\left|e_{1}\right|$ with parameter uncertainties and measurement noise. (d): Signal deviations $\left|e_{2}\right|$ with parameter uncertainties and measurement noise.

Can be seen in Figure 5 the control (CLFO) proved to be most indicated for case of the control was not subject to uncertainties. In the case of the control subject to uncertainties SDRE control proved to be indicated.

\section{CONCLUSIONS}

Two control strategies were used, suppressing the chaotic trajectory and leading the system to a desired periodic orbit, obtained by the application of the multiple scales method. A comparison of the obtained results showed that both controls are efficient. An interesting contribution of these controls is that they do not need linearization or lose the nonlinearity of the considered systems and show the robustness of the controls when the system has measurement noise.

\section{ACKNOWLEDGMENTS}

The authors thank FAPESP and $\mathrm{CNPq}$ for the financial support.

\section{REFERENCES}

1. A. Luo, F.Y. Wang, "Chaotic motion in a micro-electromechanical system with non-linearity from capacitors", Commun Nonlinear Sci Numer Simul 7, 31-49 (2002).

2. S. Liu, A. Davidson, Q. Lin, "Simulation studies on nonlinear dynamics and chaos in a MEMS cantilever control system", J Micromechan Microeng 14,10641073 (2004).

3. F. R. Chavarette, J. M. Balthazar, J. L. P. Felix, M. Rafikov, "A reducing of a chaotic movement to a periodic orbit of a micro-electro-mechanical system by using an optimal linear control design", Commun Nonlinear Sci Numer Simul 14,1844-1853 (2009).

4. A. M. Tusset, J. M. Balthazar, D. G. Bassinello, B. R. Pontes JR, J. L. P. Felix, "Statements on chaos control designs, including a fractional order dynamical system, applied to a "MEMS" comb-drive actuator", Nonlinear Dynamics, 1-21 (2012).

5. H. S. Haghighi, A. H. D. Markazi, "Chaos prediction and control in MEMS resonators", Commun Nonlinear Sci Numer Simulat 15, 3091-3099 (2010).

6. H. T. Yau, C. C. Wang, C. T. Hsieh, C. C. Cho, "Nonlinear analysis and control of the uncertain microelectro-mechanical system by using a fuzzy sliding mode control design", Computers and Mathematics with Applications 61, 1912-1916 (2011).

7. J. D. Pearson, "Approximation methods in optimal control", Journal of Electronics and Control, 13, 453469 (1962).

8. A. Wernli, G. Cook, "Suboptimal control for the nonlinear quadratic regulator problem", Automatica 11, 75-84 (1975).

9. C. P. Mracek, J. R. Cloutier, "Control designs for the nonlinear benchmark problem via the state-dependent Riccati equation method", International Journal of Robust and Nonlinear Control 8, 401-433 (1998).

10. B. Friedland, "Advanced Control System Design", Prentice-Hall, Englewood Cliffs NJ, 110-112 (1996).

11. A. Fenili, J. M. Balthazar, "The rigid-flexible nonlinear robotic manipulator: Modeling and control", Communications in Nonlinear Science \& Numerical Simulation 16, 2332-2341 (2011).

12. M. Rafikov, J. M. Balthazar, "On an optimal control design for Rössler system”, Phys. Lett. A 333, 241-245 (2004) 
13. A. M. Tusset, M. Rafikov, J. M. Balthazar, "An Intelligent controller design for magnetorheological damper based on quarter-car model", Journal of Vibration and Control 15(12), 1907-1920 (2009).

14. A. M. Tusset, J. M. Balthazar, F. R. Chavarette, J. L. P. Felix, "On energy transfer phenomena, in a nonlinear ideal and nonideal essential vibrating systems, coupled to a (MR) magneto-rheological damper", Nonlinear Dynamics, 1-22 (2012).

15. R. M. C. Mestrom, R. H. B. Fey, , J. T. M. van Beek, K. L. Phan, and H. Nijmeijer, "Modeling the dynamics of a MEMS resonator", Simulations and experiments. Sens Actuators A 142(3), 06 -15 (2007).

16. A. H. Nayfeh, B. Balachandran, "Applied Nonlinear Dynamics - Analytical, Computational, and Experimental Methods". John Wiley and Sons, (1995).

17. R. L. Burden, J. D. Faires, "Numerical analysis", Cengage Learning, (2007).

18. M. J. Shirazi, R. Vatankhah, M. Boroushaki, H. Salarieh, A. Alasty, "Application of particle swarm optimization in chaos synchronization in noisy environment in presence of unknown parameter uncertainty", Communications in Nonlinear Science and Numerical Simulation, 17(2), 742-753 (2012). 
Copyright of AIP Conference Proceedings is the property of American Institute of Physics and its content may not be copied or emailed to multiple sites or posted to a listserv without the copyright holder's express written permission. However, users may print, download, or email articles for individual use. 